\title{
Drug Reaction with Eosinophilia and Systemic Symptoms (DRESS): A Case Report
}

\author{
Islam A. M. Abu-Naylal ${ }^{1}$ and Abdulameer M. Abu Nailah ${ }^{2}$
}

\begin{abstract}
DRESS syndrome is a rare, potentially life-threatening, drug-induced hypersensitivity reaction. It presents as a skin eruption, hematologic abnormalities, lymphadenopathy and internal organ involvement (liver, kidney, lung).

We reported a case of DRESS syndrome where the patient presented with fatigue, headache, joint pain, high fever, chills, puffiness of the face and ears with diffuse rash.
\end{abstract}

Keywords: Drug reaction with Eosinophilia and systemic symptoms, DRESS, Eosinophilia, Drug-induced, hypersensitivity, Anti-convulsant.

\footnotetext{
1 Junior Trainee in Surgery, Addenbrookes Hospital, Cambridge, UK.

${ }^{2}$ Specialist Rheumatologist MBChB, F.I.C.M, F.I.C.M.S Rheumatology and medicine rehabilitation, MRCP (Rheumatology) Canadian Specialist Hospital, Dubai, UAE.
} 


\section{Introduction}

Drug reaction with eosinophilia and systemic symptoms (DRESS) is a rare, potentially life-threatening, drug-induced hypersensitivity reaction that includes skin eruption, hematologic abnormalities (eosinophilia, atypical lymphocytosis), lymphadenopathy and internal organ involvement (liver, kidney, lung). DRESS syndrome is characterized by a long latency (two to eight weeks) between drug exposure and disease onset, a prolonged course with frequent relapses despite the discontinuation of the culprit drug and frequent association with the reactivation of latent human herpesvirus infections. Most cases occur in adults without any sex predilection.

\section{Case Report}

A 30 years old Sri Lankan male patient was diagnosed with seropositive RA. On 25th February 2016, he was started on Hydroxychloroquine, Sulfasalazine (gradually increased the dose from 500mg to $2000 \mathrm{mg}$ per day) and prednisolone $5 \mathrm{mg}$ daily. After 2 weeks, the patient reported fatigue, headache and joint pain. However, his blood tests were normal apart from elevated C- Reactive Protein (CRP). A month after the initial diagnosis (26th March 2016) the patient presented with high fever, chills, cervical lymphadenopathy, generalized fatigue, vomiting and puffiness of the face and ears with diffuse skin rash (symmetrically distributed maculo-papular eruption with induration and plaque formation. The eruption later progressed to confluent lesions, erythroderma, and finally to generalized exfoliative, erythematous lesion) over the face, neck, upper back, upper chest wall and both arms, without involvement of the oral mucosa, palms, or soles. 


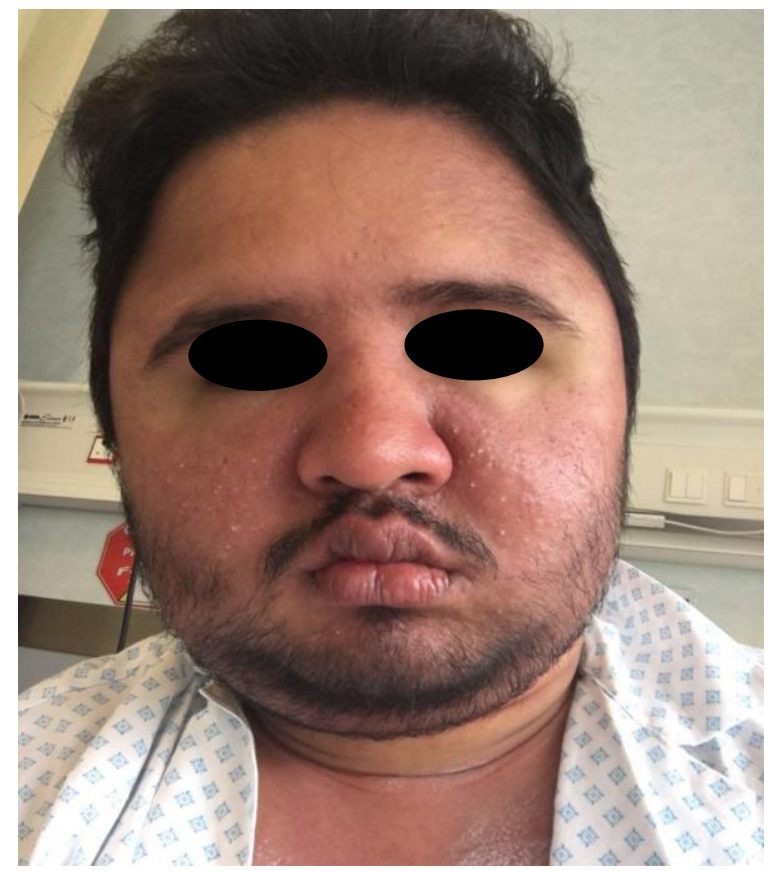

Figure 1: Patient presenting with puffy face and diffuse skin rash

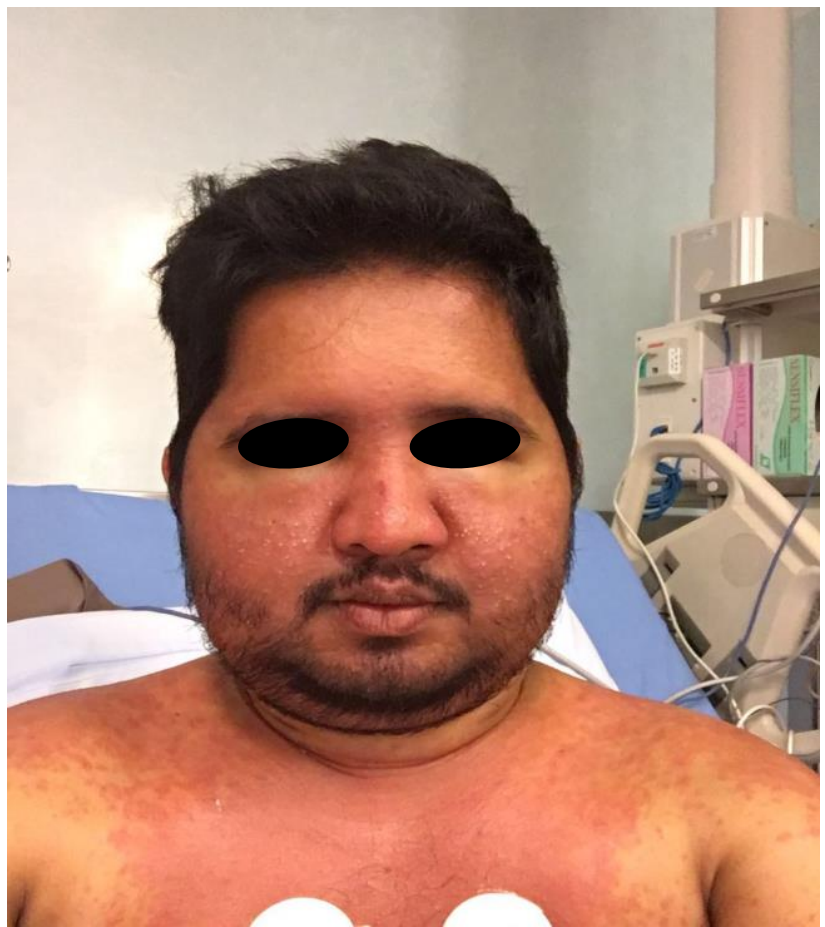

Figure 2: Patient presenting with puffy face and diffuse skin rash 
The patient had no other significant past medical history, or drug allergies. Review of systems was positive for non-productive cough, and negative for chest pain, shortness of breath, abdominal pain, or any recent altered mental status.

The patient's initial laboratory test results (performed on $25^{\text {th }}$ February 2016) showed a negative Anti-Nuclear Antibody (ANA) profile. The laboratory test results showed a CRP of 7.5, an Erythrocyte Sedimentation Rate (ESR) of 12, the Rheumatoid Factor (RF) was 37.9. Complete blood count shows that the White Blood Cells (WBC) was 7.2, Eosinophils level was 0.2, the Haemoglobin level was 14.2 and the platelet count was 260. The Alanine Aminotransferase (ALT) level was 23, the Aspartate Transferase (AST) level was 20, Creatinine level was 0.8, and the Anticyclic Citrullinated Peptide (anti-CCP) level was 7.0.

The patient's laboratory tests a month later (26 ${ }^{\text {th }}$ March 2016) after presenting with DRESS syndrome showed a normal stool analysis, negative clostridium difficile toxin in faeces. A complete blood count test also showed that the WBC level was 10.2, Eosinophil level was 1.49, platelet level was 223 and HB level was 13.3. The CRP level went from 31.70 to 62.6, the ALT level from 313 to 464, the AST level from 250 to 407, the Alkaline Phosphate was 313, the Creatinine level was 1.01, Prothrombin Time/International Normalised Ratio (PT/INR) was 1.2, the Bilirubin total was 2.776 while direct Bilirubin was 2.304, the Gamma Glutamyl transferase (GGT) level was 430, the Protein/Creatinine ratio of urine was 0.2 , the total Protein level was 5.25 and the Albumin in serum level was 3.25.

The patient's blood test results were negative for Anti-Smooth muscle antibodies, Anti-Mitochondrial antibodies, Widal test, HLA B27, Epstein Barr Virus IgM, Cytomegalovirus IgM, Measles virus IgM antibodies, Brucella IgM antibodies and Anti-Neutrophilic Cytoplasmic Antibody level (ANCA). The blood and urine culture showed no growth. Immunoglobulin (IgA, IgM \& IgG) levels were normal. The test was non-reactive to Hepatitis C Antibodies and Hepatitis B surface Antigen. The throat swab test performed for Streptococcus group A was also negative.

\section{Table 1: Peripheral smear exam results}

\section{PERIPHERAL SMEAR EXAM, EDTA WHOLE BLOQD \\ $\mathrm{RBC}$ \\ WBC}

PLATELETS

IMPRESSION
NORMOCYTIC AND NORMOCHROMIC.

EXCESS OF EOSINOPHILS AND ATYPICAL MONONUCLEAR CELLS. THESE CELLS HAVE MODERATE BASOPHILIC CYTOPLASM, THE NUCLEAR CHROMATIN SHOWS FOCAL CLUMPING SOME OF THEM HAVE PLASMACYTOID FEATURES.

ADEQUATE,

ATYPICAL MONONUCLEAR CELLS. (SEE COMMENT) 
Imaging test results showed a normal abdominopelvic Ultrasound results in the initial diagnosis. The abdominopelvic Ultrasound results of the patient after presenting with DRESS showed mild splenomegaly and less fluid in the peritoneal space inter-loop and Hepato-renal pouch. The chest X-ray result was normal.

Corticosteroid treatment was started after exclusion of other potentially serious conditions including infections. A dramatic improvement was observed and his clinical and laboratory findings were recovered on the fourth day post-treatment. At first, Methylprednisolone was given Intravenously $500 \mathrm{mg}$ once daily for 5 days, then shifted to prednisolone 30mg per mouth. The dose of Glucocorticoids could not be lowered because the rash started to recur again after attempting to taper the dose.

\section{Conclusions}

DRESS syndrome is an often under-diagnosed and under-recognized severe type IV (delayed type) hypersensitivity reaction that can occur with any medication but most commonly in response to aromatic anticonvulsants. Like most severe allergic reactions, DRESS syndrome involves rash, diffuse swelling, as well as eosinophilia. The hallmark of DRESS syndrome, however, is the presence of systemic manifestations such as inflammation of the liver, kidneys, heart, or other organs.

Although the true incidence is unknown, DRESS syndrome has been estimated to occur in approximately one out of 1000 to 10,000 new users of anti-epileptic medications and is more commonly reported in African-American men. Vitamin D deficiency has been implicated as a possible contributor to the pathogenesis of DRESS, due to its protective effects against inflammatory and auto-immune conditions and because vitamin D deficiency occurs more frequently in people with darker skin phenotypes.

Aetiology and risk factors of DRESS include, Antiepileptic agents (like Carbamazepine, Lamotrigine, Phenytoin and Phenobarbital), Allopurinol, Antipsychotic drugs like Olanzapine, Sulphonamides (particularly sulfasalazine) and Dapsone, Minocycline, Vancomycin and Kinase inhibitors, including imatinib. Pharmacogenetic studies have found an association between HLA haplotypes and susceptibility to DRESS.

The diagnosis is made by assessing history to a high-risk medication particularly. Presenting with morbilliform eruption progressing to confluent and infiltrated erythema or exfoliative dermatitis, hematologic abnormalities, systemic symptoms and organ involvement are also part of the main diagnostic criteria.

Laboratory abnormalities noted in this case include Leucocytosis with eosinophil counts of more than 700/microliter (50 to 90\%). Atypical lymphocytosis with large activated lymphocytes, lymphoblasts, or mononucleosis-like cells was also noted (30 to $70 \%$ ). There was also increased serum alanine aminotransferase found (up to $80 \%$ ). An HHV-6 infection (40 to $60 \%$ ) was also found. There was also a moderate increase in creatinine level and low-grade proteinuria. 
So far, prompt withdrawal of the offending drug is the only undisputed way to treat drug hypersensitivity reactions. Supportive therapy includes antipyretics and the use of topical steroids to improve symptoms. Systemic corticosteroids can reduce symptoms of delayed hypersensitivity reactions.

They are known to inhibit the effect of interleukin-5 on eosinophils accumulation occurring in this syndrome, which may explain their benefit in the treatment. Dramatic improvement in clinical symptoms and laboratory findings has been observed soon after the beginning of corticoid therapy in independent case reports. Relapses have been described after tapering or withdrawal of systemic steroids. Mortality rate in DRESS syndrome is about $10 \%$, mostly due to liver failure. The prognosis is mostly good with most patients making complete recovery in weeks or a few months after drug withdrawal. Autoimmune diseases have been reported in some patients, months or years after the resolution of the drug reaction.

ACKNOWLEDGEMENTS: We thank the patient and his family members for providing consent and for his participation in the case. 


\section{References}

[1] Bocquet H, Bagot M and Roujeau JC (1996) Drug-induced pseudolymphoma and drug hypersensitivity syndrome (Drug Rash with Eosinophilia and Systemic Symptoms: DRESS). Semin Cutan Med Surg, 15: 250-257. 10.1016/S1085-5629(96)80038-1.

[2] Shiohara T, Iijima M, Ikezawa Z, et al (2007) The diagnosis of DRESS syndrome has been sufficiently established on the basis of typical clinical features and viral reactivations. Response Br J Dermatol, 156: 1045-1092. 10.1111/j.1365-2133.2007.07775.x.

[3] Criado PR, Criado RF, Avancini M, et al (2012) Drug reaction with Eosinophilia and Systemic Symptoms (DRESS) / Drug-induced Hypersensitivity Syndrome (DIHS): a review of current concepts. An Bras Dermatol, 87 (3): 435-449. 10.1590/S0365-05962012000300013.

[4] Carroll MC, Yueng-Yue KA, Esterly NB, et al (2001) Drug-induced hypersensitivity syndrome in pediatric patients. Pediatrics, 108 (2): 485-492. 10.1542/peds.108.2.485.

[5] Ben m'rad M, Leclerc-Mercier S, Blanche P, et al (2009) Drug-induced hypersensitivity syndrome: clinical and biologic disease patterns in 24 patients. Medicine (Baltimore), 88: 131-140. 10.1097/MD.0b013e3181a4d1a1.

[6] Cacoub P, Musette P, Descamps V, et al (2011) The DRESS syndrome: a literature overview. Am J Med, 124 (7): 588-597.

10.1016/j.amjmed.2011.01.017.

[7] Frieling G, Jessup C and Mihm M (2012) Drug rash with eosinophilia and systemic symptoms. Diagnostic Pathology: Non-neoplastic Dermatopathology, 10-11. 1

[8] Chaiken BH, Goldberg BI and Segal JP (1950) Dilantin sensitivity; report of a case of hepatitis with jaundice, pyrexia and exfoliative dermatitis. N Engl J Med, 242 (23): 897-898. 10.1056/NEJM195006082422304.

[9] Kardaun SH, Sidoroff A, Valeyrie-Allanore L, et al (2007) Variability in the clinical pattern of cutaneous side-effects of drugs with systemic symptoms: does a DRESS syndrome really exist?. Br J Dermatol, 156 (3): 609-611. 10.1111/j.1365-2133.2006.07704.x.

[10] Tennis P and Stem RS (1997) Risk of serious cutaneous disorders after initiation of use of phenytoin, carbamazepine, or sodium valproate: a record linkage study. Neurology, 49: 542-546. 10.1212/WNL.49.2.542.

[11] Carroll MC, Yueng-Yue KA, Esterly NB, et al (2001) Drug-induced hypersensitivity syndrome in pediatric patients. Pediatrics. 108 (2): 485-492. 10.1542/peds.108.2.485.

[12] Sullivan JR and Shear NH. (2001) The drug hypersensitivity syndrome: what is the pathogenesis? Arch Dermatol, 137:357-64

[13] Knowles S, Shapiro L and Shear NH (1997) Serious dermatologic reactions in children. Curr Opin Pediatr, 9:388-95 
[14] Hellman C, Lönnkvist K, Hedlin G, et al (2002) Down-regulated IL-5 receptor expression on peripheral blood eosinophils from budesonide-treated children with asthma. Allergy, 57:323-8

[15] Chopra S, Levell NJ, Cowley G, et al (1996) Systemic corticosteroids in the phenytoin hypersensitivity syndrome. Br J Dermatol, 134:1109-12

[16] Tas S and Simonart T. (1999) Drug rash with eosinophilia and systemic symptoms (DRESS syndrome). Acta Clin Belg, 54:197-200

[17] Roujeau JC. (1999) Treatment of severe drug eruptions. J Dermatol, 26:71822

[18] Callot V, Roujeau JC, Bagot M, et al (1996) Drug-induced pseudolymphoma and hypersensitivity syndrome. Two different clinical entities. Arch Dermatol, 132:1315-21 